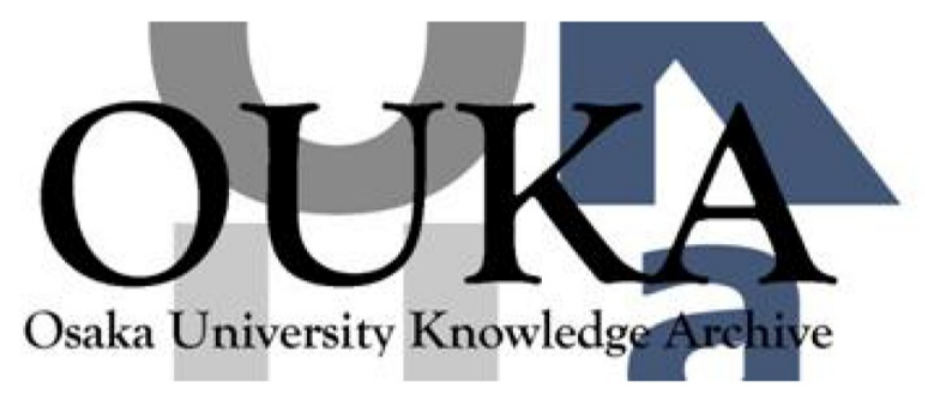

\begin{tabular}{|c|l|}
\hline Title & Quantum Spin-Hall Effect in a Quantum Wire \\
\hline Author(s) & Hattori, Kiminori \\
\hline Citation & $\begin{array}{l}\text { Journal of the Physical Society of Japan. } \\
79(10) \text { p. 104709-1-p. 104709-5 }\end{array}$ \\
\hline Issue Date & $2010-10$ \\
\hline oaire:version & AM \\
\hline URL & https://hdl. handle. net/11094/3413 \\
\hline rights & \\
\hline Note & \\
\hline
\end{tabular}

Osaka University Knowledge Archive : OUKA

https://ir. Library. osaka-u. ac. jp/

Osaka University 


\title{
Quantum Spin-Hall Effect in a Quantum Wire
}

\author{
Kiminori HATTORI* \\ Department of Systems Innovation, Graduate School of Engineering Science, Osaka University, \\ Toyonaka, Osaka 560-8531, Japan
}

In this paper, we show that spatially displaced helical states that transport electrons with opposite spins in opposite directions are formed in a harmonic quantum wire due to the spin-orbit (SO) coupling induced by lateral confinement. These states give rise to a transverse spin imbalance in response to the longitudinal charge current as well as a transverse charge imbalance in response to the longitudinal spin current. We analyze the consequential direct and inverse spin-Hall effects in terms of the spin-charge reciprocity relations in a unified manner by virtue of spin conservation. Through a numerical calculation based on the nonequilibrium Green's function formalism, we demonstrate that a generalized spin-Hall resistance that identically describes charge-to-spin and spin-to-charge conversions exhibits a series of quantum plateaus when the SO coupling is sufficiently strong.

Keywords: quantum spin-Hall effect, quantum wire, charge-to-spin conversion, spin-to-charge conversion, nonequilibrium Green’s function

*E-mail: hattori@ee.es.osaka-u.ac.jp 


\section{Introduction}

The quantum Hall (QH) effect occurs when a two-dimensional (2D) system of electrons is subject to a strong magnetic field perpendicular to the $2 \mathrm{D}$ plane. ${ }^{1)}$ In the $\mathrm{QH}$ state, the Hall resistance is intermittently stuck at quantized values for a finite range of control parameter, and depends only on how many edge modes contribute to electrical conduction. ${ }^{2)}$ The edge mode is chiral in nature since it carries a charge current unidirectionally along the perimeter of the system, reflecting the broken time-reversal symmetry due to the magnetic field. Recently, a spin analog to the conventional QH effect has been theoretically predicted in time-reversal symmetric situations for a class of spin-orbit (SO) coupled systems, such as a single sheet of graphene with an intrinsic SO coupling, ${ }^{3)}$ an inverted HgTe/CdTe quantum well structure, ${ }^{4,5)}$ as well as a zinc-blende semiconductor with a shear strain gradient. ${ }^{6}$ In these systems, helical edge modes emerge that transport electrons with opposite spins in opposite directions, exhibiting a phenomenology called the quantum spin-Hall (QSH) effect. ${ }^{7,8)}$ In the simplest view, the QSH effect is regarded as two copies of the QH effect with different spins arranged so that time-reversal symmetry is preserved. The charge-Hall resistance in each spin subsystem is quantized but with the opposite sign for the opposite spin because of the helical property of transport channels. Consequently, the charge-Hall resistance totally vanishes in the whole system while the nonzero and quantized spin-Hall resistance persists. The QSH effect attracts a tremendous amount of interest in the field of condensed matter physics because it not only introduces a novel quantum state of matter but also allows electric manipulation of the spin degrees of freedom without the use of magnetic materials and magnetic fields.

In this paper, we investigate the possibility of the QSH effect in a mesoscopic quantum wire 
(QW). In this system, the helical modes are formed due to the SO coupling induced by lateral electrostatic confinement, giving rise to a transverse spin imbalance in response to the longitudinal charge current as well as a transverse charge imbalance in response to the longitudinal spin current. The resulting direct and inverse spin-Hall effects are numerically analyzed using the nonequilibrium Green's function formalism. In our previous study, ${ }^{9)}$ we argued for the occurrence of a spin-Hall current in a four-terminal junction of such QWs. In the present study, we primarily examine a spin-Hall voltage between two Hall probes attached to a single QW, and demonstrate that the QSH effect should be observable in this configuration for a strong SO coupling.

\section{Theoretical Analysis}

Let us consider a 2D electron gas in the $x y$ plane subjected to an electrostatic potential $U(x, y)$. This system is described by the single-particle Hamiltonian including the SO coupling due to the in-plane potential gradient: $H=\mathbf{p}^{2} / 2 m-(\lambda / h)\left(\mathbf{e}_{z} \times \nabla U\right) \cdot \mathbf{p} \sigma_{z}+U$, where $\mathbf{p}$ is the canonical momentum, $m$ is the electron mass, $\sigma_{z}$ is the Pauli spin matrix, $\lambda$ is the SO coupling strength, and $\mathbf{e}_{z}$ is the unit vector in the $z$ direction. ${ }^{9-16)}$ The Hamiltonian $H$ commutes with $\sigma_{z}$, and is diagonal in spin space such that $H_{\sigma \sigma^{\prime}}=H_{\sigma} \delta_{\sigma \sigma^{\prime}}$. It is informative to arrange $H_{\sigma}$ into the form

$$
H_{\sigma}=\frac{1}{2 m}(\mathbf{p}-e \sigma \mathbf{A})^{2}+U_{e f f},
$$

where $U_{\text {eff }}=U-\left(m \lambda^{2} / 2 h^{2}\right)(\nabla U)^{2}$ represents the effective electrostatic potential, and $\sigma= \pm 1$ for

spin- $\uparrow$ and $\downarrow$ states, respectively. The in-plane vector potential given by $\mathbf{A}=(m \lambda / e h) \mathbf{e}_{z} \times \nabla U$ defines the relevant out-of-plane magnetic field $\mathbf{B}=\nabla \times \mathbf{A}=\mathbf{e}_{z}(m \lambda / e h) \nabla^{2} U$. Thus, the lateral SO coupling exerts the internal magnetic fields $\sigma \mathbf{B}$ on electrons with opposite spins in opposite 
directions, and thereby generates a spin-dependent orbital effect.

A simple example of $U(x, y)$ is a 1D harmonic potential $U=\frac{1}{2} m \omega_{0}^{2} y^{2}$, which is realizable in a narrow QW running in the $x$ direction. In this case, the vector potential is written as $\mathbf{A}=B(-y, 0,0)$, where $B=\eta\left(m \omega_{0} / e\right), \quad \eta=\lambda / I^{2}$, and $\mathrm{I}=\sqrt{\mathrm{h} / m \omega_{0}}$. This expression is formally identical to the Landau gauge. The Landau-gauge Hamiltonian is translationally invariant along the $x$-axis, and hence the momentum $p_{x}$ becomes a conserved quantity. The symmetric gauge described by $\mathbf{A}=(B / 2)(-y, x, 0)$ and $B=2 \eta\left(m \omega_{0} / e\right)$ is derived for an isotropic $2 \mathrm{D}$ harmonic potential $U=\frac{1}{2} m \omega_{0}^{2}\left(x^{2}+y^{2}\right)$, which is frequently employed to model a quantum dot or disk. The symmetric-gauge Hamiltonian is rotationally invariant about the $z$-axis so that the orbital angular momentum $L_{z}=x p_{y}-y p_{x}$ is conserved. In both cases, the effective electrostatic potential is given by $U_{\text {eff }}=\left(1-\eta^{2}\right) U$, indicating that the lateral confinement is substantially reduced for a strong SO coupling. It is notable that $\eta=1$ is a special point where $U_{\text {eff }}$ completely vanishes. The problem we address then becomes equivalent to the spin-dependent Landau quantization of unconfined 2D electrons. Apparently, this picture is the same as that employed to illustrate the QSH effect due to a strain gradient. ${ }^{6)}$ In the following, we focus our attention on a harmonic QW. This is an interesting open system feasible for charge and spin injections.

The Schrödinger equation $H_{\sigma} \psi_{N Y \sigma}=\varepsilon_{N Y} \psi_{N Y \sigma}$ is analytically solved for $U=\frac{1}{2} m \omega_{0}{ }^{2} y^{2}$. The solutions are expressed as

$$
\begin{aligned}
& \psi_{N Y \sigma}(x, y)=\frac{1}{\sqrt{L}} \exp (i k x) \chi_{N}(y-\sigma Y), \\
& \varepsilon_{N Y}=\mathrm{h} \omega_{0}\left(N+\frac{1}{2}\right)+\eta^{-2} U_{e f f}(Y),
\end{aligned}
$$

where $N=0,1,2, \mathrm{~L}$ represents the subband index, $\chi_{N}(y)$ denotes the $N$ th eigenfunction of the 
1D harmonic oscillator, $k$ stands for the propagation wave vector along $x$, and $Y=-\lambda k$ is the specific $y$-coordinate mapped onto $k$. The spatial displacement of the eigenfunction by an amount of $\sigma Y$ is reasonably interpreted in terms of a spin-dependent Lorentz-type force due to the lateral SO coupling. ${ }^{9)} \quad$ As indicated by eqs. (2) and (3), there exists a fourfold degeneracy in each subband, which is closely related to time-reversal and space-inversion symmetries of the present model. The doubly degenerate states belonging to the same center coordinate $\sigma Y$ constitute a Kramers pair, which transports electrons with opposite spins in opposite directions. These helical modes or chiral spin channels carry a spin current unidirectionally. The inversion symmetry ensures that oppositely propagating spin channels are symmetrically placed around $y=0$. For $\eta<1$, each subband exhibits a parabolic dispersion reflecting the effective confining potential $U_{\text {eff }}$. Recall that $U_{\text {eff }}=0$ when $\eta=1$. Then, the subband dispersion disappears, leaving discrete bulk Landau levels spaced by $\mathrm{h} \omega_{0}$. The predicted features are clearly found in Fig. 1, which displays the energy spectra of a QW with a finite width $W$ obtained from numerical diagonalization. This figure also explains that edge modes providing gapless excitations emerge near transverse boundaries of a finite-width QW. ${ }^{17)}$

Here, we briefly discuss an elemental charge-Hall effect occurring in a spin subsystem where a couple of forward and backward propagating channels exists per one subband. The spin-resolved $\mathrm{QH}$ effect is instructive for understanding the QSH effect since it is basically comprised of cooperative QH effects for up and down spins. In terms of the Heisenberg equation, the velocity operator is defined by $\mathbf{v}^{\sigma}=(\mathbf{p}-e \sigma \mathbf{A}) / m$ for each spin state, leading to the expectation value of longitudinal velocity $\left\langle N Y \sigma\left|v_{x}^{\sigma}\right| N Y \sigma\right\rangle=-(\lambda / \mathrm{h}) \partial \varepsilon_{N Y} / \partial Y$ proportional to the slope of subband dispersion. When oppositely propagating states on opposite sides are unequally filled with electrons up to the respective chemical potentials $\mu_{\sigma}^{( \pm)}$at $y= \pm W / 2$, the longitudinal particle 
current follows the relation $J_{x}^{\sigma}=-\sigma v\left(\mu_{\sigma}^{(+)}-\mu_{\sigma}^{(-)}\right) / h$, where $v$ is the number of occupied subbands. This accounts for the quantized Hall resistance $R_{H}^{\sigma}=V_{y}^{\sigma} / I_{x}^{\sigma}=-\sigma h / v e^{2}$, where $I_{x}^{\sigma}=e J_{x}^{\sigma}$ is the longitudinal electric current, and $V_{y}^{\sigma}=\left(\mu_{\sigma}^{(+)}-\mu_{\sigma}^{(-)}\right) / e$ is the transverse Hall voltage. Except for the distinct spin dependence of $R_{H}^{\sigma}$, the straightforward explanation given above is analogous to the edge-current picture of the ordinary QH effect. ${ }^{1,2)}$ However, this picture breaks down when the oppositely propagating states spatially overlap with each other, and each interacts with both boundaries with finite probabilities. $^{18,19)}$ In such a case, the Hall resistance formula is generalized into $R_{H}^{\sigma}=-\sigma F h / v e^{2}$ with $F=\sum_{N}\left(p_{N}-q_{N}\right) / \sum_{N}\left(p_{N}+q_{N}\right)$. Here, $p_{N}\left(q_{N}\right)$ represents the transition probability of an electron between the $N$ th channel and the Hall probe on the same (opposite) side, and the summation is taken over occupied subbands. The form factor $F$ explains that $R_{H}^{\sigma}$ is no longer quantized unless $p_{N}>q_{N}$. The spatial separation between two counterpropagating channels amounts to $\Delta Y=2 \mathrm{l} / \sqrt{\eta^{-2}-1}$ at the midpoint between adjacent subband bottoms, implying that the QSH effect tends to be quenched when $\eta<<1$.

We next proceed to a more quantitative analysis of the spin-Hall effect in a four-terminal configuration based on the nonequilibrium Green’s function formalism. ${ }^{20,21)}$ The model used for the calculation consists of an infinitely-long harmonic QW of a width $W$ and two voltage probes of the same width $L$ attached symmetrically on the transverse ends of the QW (see, the inset of Fig. 2). The current leads (labeled by 1 and 3) are part of the QW in which the lateral SO coupling is present, while the confinement potential and the SO interaction are assumed to be absent inside the voltage leads (labeled by 2 and 4). In the tight-binding representation on a square lattice with lattice spacing $a$, the Hamiltonian describing the QW reads

$$
H_{\sigma}=\sum_{\mathbf{r}, \mathbf{r}^{\prime}} t_{\mathbf{r r}^{\prime}}^{\sigma} C_{\mathbf{r} \sigma}^{\dagger} C_{\mathbf{r}^{\prime} \sigma}
$$




$$
t_{\mathbf{r r}^{\prime}}^{\sigma}= \begin{cases}-t\left(1+i \sigma \phi_{\mathbf{r r}^{\prime}}\right), & \mathbf{r}=\mathbf{r}^{\prime} \pm a \mathbf{e}_{x, y} \\ 4 t+U, & \mathbf{r}=\mathbf{r}^{\prime} \\ 0, & \text { otherwise, }\end{cases}
$$

where $c_{\mathbf{r} \sigma}\left(c_{\mathbf{r} \sigma}^{\dagger}\right)$ is the annihilation (creation) operator of an electron at position $\mathbf{r}$ with spin $\sigma$, $t=\mathrm{h}^{2} / 2 m a^{2}$ is the hopping energy, and $\phi_{\mathbf{r r}^{\prime}}=(e / \mathrm{h}) \mathbf{A} \cdot\left(\mathbf{r}-\mathbf{r}^{\prime}\right)$ is the Aharonov-Bohm phase for a path connecting two nearest neighbors. The Peierls substitution (by which $1+i \sigma \phi_{\mathrm{rr}^{\prime}} \rightarrow e^{i \sigma \phi_{\mathrm{rr}^{\prime}}}$ and $U \rightarrow U_{\text {eff }}$ ) is not employed in eq. (5), being consistent with the original Hamiltonian, which contains the SO coupling term linear in A. Using the multiterminal Landauer-Büttiker formula, the spin-resolved particle current in lead $\alpha$ is expressed as $J_{\alpha \sigma}=h^{-1} \sum_{\beta} T_{\alpha \beta}^{\sigma}\left(\mu_{\alpha \sigma}-\mu_{\beta \sigma}\right)$, where $\mu_{\alpha \sigma}$ is the spin-dependent chemical potential. The spin-resolved transmission coefficient is defined by $T_{\alpha \beta}^{\sigma}=\operatorname{Tr}\left(\Gamma_{\alpha \sigma} G_{\sigma}^{R} \Gamma_{\beta \sigma} G_{\sigma}^{A}\right)$, where $G_{\sigma}^{R(A)}(\varepsilon)=\left[\varepsilon-H_{\sigma}-\sum_{\alpha} \sum_{\alpha \sigma}^{R(A)}\right]^{-1}$ is the retarded (advanced) Green's function, $\Sigma_{\alpha \sigma}^{R(A)}$ is the retarded (advanced) self-energy, and $\Gamma_{\alpha \sigma}=i\left(\Sigma_{\alpha \sigma}^{R}-\Sigma_{\alpha \sigma}^{A}\right)$. Following the Keldysh formulation, the lesser Green's function is represented as $G_{\sigma}^{<}=i \sum_{\alpha} f_{\alpha \sigma} A_{\alpha \sigma}$, where $A_{\alpha \sigma}=G_{\sigma}^{R} \Gamma_{\alpha \sigma} G_{\sigma}^{A}$ is the spectral function, and $f_{\alpha \sigma}(\varepsilon)=f\left(\varepsilon-\mu_{\alpha \sigma}\right)$ is the Fermi-Dirac distribution function. The diagonal element of $G_{\sigma}^{<}$in position space is directly related to the particle density $\rho_{\sigma}(\mathbf{r})=a^{-2}\left\langle c_{\mathbf{r} \sigma}^{\dagger} c_{\mathbf{r} \sigma}\right\rangle$. The deviation of $\rho_{\sigma}(\mathbf{r})$ from its equilibrium value is simply given by $\delta \rho_{\sigma}(\mathbf{r})=\left(2 \pi a^{2}\right)^{-1} \sum_{\alpha}\left(A_{\alpha \sigma}\right)_{\mathbf{r r}} \delta \mu_{\alpha \sigma}$ for a sufficiently small variation of chemical potential $\delta \mu_{\alpha \sigma}$. Note that all of these formulae assume spin conservation.

It is appropriate here to introduce the notations $Q^{ \pm}=\left(Q_{\uparrow} \pm Q_{\downarrow}\right) / 2$ symmetrically defining charge $\left(Q^{+}\right)$and spin $\left(Q^{-}\right)$components of a physical quantity $Q$, which are useful in explicitly formulating the spin-charge transformation for spin-conserving systems. The charge and spin currents $J_{\alpha}^{ \pm}$can be written as

$$
J_{\alpha}^{ \pm}=\frac{1}{h} \sum_{\beta}\left[T_{\alpha \beta}^{+}\left(\mu_{\alpha}^{ \pm}-\mu_{\beta}^{ \pm}\right)+T_{\alpha \beta}^{-}\left(\mu_{\alpha}^{\mathrm{m}}-\mu_{\beta}^{\mathrm{m}}\right)\right]
$$

It is evident from eq. (6) that $J^{ \pm}$is transformed into $J^{\mathrm{m}}$ by replacing $\mu^{ \pm}$with $\mu^{\mathrm{m}}$. A similar 
transformation holds between the charge and spin densities $\delta \rho^{ \pm}$expressed as

$$
\delta \rho^{ \pm}(\mathbf{r})=\frac{1}{2 \pi a^{2}} \sum_{\alpha}\left[\left(A_{\alpha}^{+}\right)_{\mathrm{rr}} \delta \mu_{\alpha}^{ \pm}+\left(A_{\alpha}^{-}\right)_{\mathrm{rr}} \delta \mu_{\alpha}^{\mathrm{m}}\right]
$$

Armed with these transformations, we analytically examine the spin-Hall phenomenology within the framework initially proposed by Hirsch. ${ }^{22)}$ In the four-terminal spin-Hall configuration, a longitudinal charge current $J_{1}^{+}=-J_{3}^{+}$driven by a charge bias $\mu_{1}^{+}-\mu_{3}^{+}$induces a transverse spin imbalance, generating a spin bias $\mu_{4}^{-}-\mu_{2}^{-}$under the condition that $J_{1}^{-}=J_{3}^{-}=0$ and $J_{2}^{ \pm}=J_{4}^{ \pm}=0$. The charge to spin conversion in this setup is described in terms of $G_{13,13}^{++}=I_{1}^{+} /\left(V_{1}^{+}-V_{3}^{+}\right)$and $R_{13,42}^{+-}=\left(V_{4}^{-}-V_{2}^{-}\right) / I_{1}^{+}$, where $I_{\alpha}^{ \pm}=e J_{\alpha}^{ \pm}$and $V_{\alpha}^{ \pm}-V_{\beta}^{ \pm}=\left(\mu_{\alpha}^{ \pm}-\mu_{\beta}^{ \pm}\right) / e$. The inverse spin-Hall configuration is prescribed contrastingly, where a longitudinal spin current $J_{1}^{-}=-J_{3}^{-}$driven by a spin bias $\mu_{1}^{-}-\mu_{3}^{-}$induces a transverse charge imbalance, generating a charge bias $\mu_{4}^{+}-\mu_{2}^{+}$under the condition that $J_{1}^{+}=J_{3}^{+}=0$ and $J_{2}^{ \pm}=J_{4}^{ \pm}=0$. In this case, the spin to charge conversion is described in terms of the $G_{13,13}^{--}=I_{1}^{-} /\left(V_{1}^{-}-V_{3}^{-}\right)$and $R_{13,42}^{-+}=\left(V_{4}^{+}-V_{2}^{+}\right) / I_{1}^{-}$. (In the context of topological field theory, ${ }^{23-25)}$ the direct and inverse spin-Hall effects are unifyingly referred to as the dual QSH effect in the quantization regime.) A quantitative equivalence between these two effects is shown by the reciprocity relations applicable to the longitudinal conductance and the spin-Hall resistance

$$
\begin{aligned}
& G_{13,13} \equiv G_{13,13}^{++}=G_{13,13}^{--}, \\
& R_{13,42} \equiv R_{13,42}^{+-}=R_{13,42}^{-+},
\end{aligned}
$$

which are readily obtained from the spin-charge transformation. An analogous relation

$$
\hat{\rho} \equiv \delta \rho^{-} /\left(\mu_{1}^{+}-\mu_{3}^{+}\right)=\delta \rho^{+} /\left(\mu_{1}^{-}-\mu_{3}^{-}\right),
$$

is also established between the spin distribution $\delta \rho^{-}$occurring in the spin-Hall configuration and the charge distribution $\delta \rho^{+}$occurring in the inverse spin-Hall configuration. For this reason, the direct and inverse spin-Hall effects can be identically treated in terms of $G_{13,13}, R_{13,42}$, as well as $\hat{\rho}$. 
It may be worthwhile stressing that eqs. (8)-(10) are not deduced from the Onsager's reciprocity theorem but are guaranteed simply by spin conservation (where charge conservation is trivially implied).

\section{Numerical Calculation}

The spin-charge reciprocity relations greatly simplify the numerical analysis of the direct and inverse spin-Hall effects. In the calculation, the energy $h \omega_{0}$ is taken as the energy unit. The hopping energy is normally set at $t=10 \mathrm{~h} \omega_{0}$, which corresponds to the length scale of the confinement $\mathrm{I} \approx 5 a$, permitting our lattice model to reasonably simulate a continuum system. Figure 2 displays the longitudinal conductance $G_{13,13}$ and the spin-Hall resistance $R_{13,42}$ calculated as a function of the Fermi energy $\mu$ for various values of the normalized SO coupling strength $\eta=\lambda / \mathrm{I}^{2}$. The longitudinal conductance $G_{13,13}$ is independent of $\eta$, and is fully quantized in units of $e^{2} / h$, indicating a formation of ballistic transport channels unperturbed by the attached Hall contacts. This property enables a noninvasive observation of the spin-Hall effect intrinsic in QW. The spin-Hall resistance $R_{13,42}$ strongly depends on $\eta$. For $\eta \approx 1, R_{13,42}$ exhibits perfect quantum plateaus at $h / v e^{2}$ (with $v=1,2,3, \mathrm{~L}$ ) characteristic of the QSH regime, while the QSH effect is suppressed when $\eta<<1$. Figure 3 shows the relation between $R_{13,42}$ and $\eta$ calculated for $\mu=\mathrm{h} \omega_{0}$, explaining that $R_{13,42}$ increases linearly with $\eta$ up to $\eta \cong 0.1$ and then saturates at the quantized value $h / e^{2}$ (for $v=1$ ) around $\eta=1$. These behaviors are reasonably accounted for by the channel separation $\Delta Y$ depending on $\eta$. Figure 4 illustrates the nonequilibrium spin/charge distribution $\hat{\rho}$ occurring at $\mu=\mathrm{h} \omega_{0}$ in the direct/inverse spin-Hall configuration. The density $\hat{\rho}$ appears with a positive sign on the lower side and a negative sign on the upper side. 
The spatial spin/charge polarization, which is the origin of the transverse spin/charge bias $\mu_{4}^{\mathrm{m}}-\mu_{2}^{\mathrm{m}}$, arises from unequally populated helical modes. The lower helical modes consist of rightward propagating spin- $\uparrow$ channels and leftward propagating spin- $\downarrow$ channels, while the upper helical modes consist of leftward propagating spin- $\uparrow$ channels and rightward propagating spin- $\downarrow$ channels. In the presence of the longitudinal charge bias $\mu_{1}^{+}-\mu_{3}^{+}$, consequently, the lower channels are populated with spin- $\uparrow$ electrons, and simultaneously the upper channels are populated with spin- $\downarrow$ electrons. On the other hand, in the presence of the spin bias $\mu_{1}^{-}-\mu_{3}^{-}$, the lower channels are populated whereas the upper channels are depopulated regardless of their spin polarizations. Because of a large channel separation in the QSH state, backscattering into oppositely propagating channels of the same spin hardly takes place even though random impurities are present. This feature has been confirmed by the numerical calculation for disordered systems (not shown).

Semiconductor QW structures can be fabricated in various ways, e.g., the split gate technique, shallow and deep mesa etchings, as well as focused-ion-beam implantation. ${ }^{26,27)}$ In the literature, ${ }^{27)}$ a level spacing as large as $\mathrm{h} \omega_{0}=7 \mathrm{meV}$ has been reported for a ballistic QW made of GaAs. The SO coupling strengths have been theoretically evaluated for some conventional semiconductors. ${ }^{28,29)}$ For example, $\lambda=0.05 \mathrm{~nm}^{2}$ for GaAs, $1 \mathrm{~nm}^{2}$ for InAs , $5 \mathrm{~nm}^{2}$ for InSb, and $20 \mathrm{~nm}^{2}$ for $\mathrm{Hg}_{0.8} \mathrm{Cd}_{0.2} \mathrm{Te}$. These parameters lead to $\eta$ ranging from $3 \times 10^{-4}$ to 0.05, suggesting that the QSH effect is not easy to realize experimentally. Nevertheless, it should be emphasized that the quantization and unquantization regimes are connected continuously, and the spin-Hall effect driven by the same mechanism is expected to occur in realistic QW systems. The transverse spin bias induced by the longitudinal charge current manifests the spin-Hall phenomenology due to the lateral SO coupling. The transverse charge bias induced by the 
longitudinal spin current is practically useful since it provides an experimental scheme for electrical detection of a pure spin flux.

\section{Summary}

In summary, we have investigated the lateral SO coupling in a harmonic QW and its application to the QSH effect. In this system, electron spin is conserved while spatially displaced helical states emerge which transport electrons with opposite spins in opposite directions. These states are unequally populated in the presence of the longitudinal charge/spin current, generating the transverse spin/charge imbalance. The resulting spin/charge bias is detectable noninvasively by the Hall probes coupled to the QW. The direct and inverse spin-Hall effects due to the lateral SO coupling are identically treated in terms of the spin-charge reciprocity relations based on spin conservation. The spatial separation between the transport channels crucially depends on the normalized SO coupling strength $\eta=\lambda / /^{2}$. A large channel separation allowed for $\eta \approx 1$ yields the quantized spin-Hall resistance whereas the quantization is suppressed when $\eta<<1$.

\section{Acknowledgement}

This work was supported by a Grant-in-Aid for Scientific Research (No. 21540320) from the Japan Society for the Promotion of Science. 


\section{REFERENCES}

1) See, e.g., D. Yoshioka: The Quantum Hall Effect (Springer-Verlag, New York, 2002).

2) M. Büttiker: Phys. Rev. B 38 (1988) 9375.

3) C. L. Kane and E. J. Mele: Phys. Rev. Lett. 95 (2005) 226801.

4) B. A. Bernevig, T. L. Hughes, and S.-C. Zhang: Science 314 (2006) 1757.

5) M. König, S. Wiedmann, C. Brüne, A. Roth, H. Buhmann, L. W. Molenkamp, X.-L. Qi, and S.-C. Zhang: Science 318 (2007) 766.

6) B. A. Bernevig and S.-C. Zhang: Phys. Rev. Lett. 96 (2006) 106802.

7) $\quad$ N. Nagaosa: Science 318 (2007) 758.

8) C. Day: Phys. Today 61 (2008) No. 1, 19.

9) K. Hattori and H. Okamoto: Phys. Rev. B 74 (2006) 155321.

10) A. V. Moroz and C. H. W. Barnes: Phys. Rev. B 60 (1999) 14272.

11) S. Bellucci and P. Onorato: Phys. Rev. B 73 (2006) 045329.

12) Y. Jiang and L. Hu: Phys. Rev. B 74 (2006) 075302.

13) Y. Xing, Q.-F. Sun, L. Tang, and J.-P. Hu: Phys. Rev. B 74 (2006) 155313.

14) M. Yamamoto and B. Kramer: J. Appl. Phys. 103 (2008) 123703.

15) T. Yokoyama and M. Eto: Phys. Rev. B 80 (2009) 125311.

16) P. Debray, S. M. S. Rahman, J. Wan, R. S. Newrock, M. Cahay, A. T. Ngo, S. E. Ulloa, S. T. Herbert, M. Muhammad, and M. Johnson: Nat. Nanotechnol. 4 (2009) 759.

17) In this calculation, the SO coupling induced by hard wall potentials is ignored, which may generate local spin-dependent vector potentials on the edges of the finite-size system. This contribution is however irrelevant because the electron wavefunction vanishes at the edges. 
18) F. M. Peeters: Phys. Rev. Lett. 61 (1988) 589.

19) H. Akera and T. Ando: Phys. Rev. B 39 (1989) 5508.

20) S. Datta: Electronic Transport in Mesoscopic Systems (Cambridge University Press, Cambridge, 1995).

21) H. Haug and A.-P. Jauho: Quantum Kinetics in Transport and Optics of Semiconductors (Springer-Verlag, New York, 2007).

22) J. E. Hirsch: Phys. Rev. Lett. 83 (1999) 183.

23) M. Freedman, C. Nayak, K. Shtengel, K. Walker, and Z. Wang: Ann. Phys. (N.Y.) 310 (2004) 428.

24) T. H. Hansson, V. Oganesyan, and S. L. Sondhi: Ann. Phys. (N.Y.) 313 (2004) 497.

25) J. Goryo and N. Maeda: arXiv:1007.1507 (to be published in J. Phys. Soc. Jpn.).

26) Mesoscopic Physics and Electronics, edited by T. Ando, Y. Arakawa, K. Furuya, S. Komiyama, and H. Nakashima (Springer-Verlag, New York, 1998).

27) Y. Hirayama and T. Saku: Appl. Phys. Lett. 54 (1989) 2556.

28) G. Lommer, F. Malcher, and U. Rössler: Phys. Rev. Lett. 60 (1988) 728.

29) E. A. de Andrada e Silva, G. C. La Rocca, and F. Bassani: Phys. Rev. B 55 (1997) 16293. 


\section{FIGURE CAPTIONS}

Fig. 1

(Color online) Energy dispersion calculated for a finite-width QW. Two panels show the energy spectra of (a) the lowest subband ( $N=0$ ) for $\eta$ varying in 0.1 steps and (b) a series of subbands $(N=0,1,2, \mathrm{~L}, 9)$ for $\eta=1$. In the calculation, a continuum limit is taken in the $x$ direction while the tight-binding approximation is adopted in the $y$ direction. The parameters used in the calculation are $t=10 \mathrm{~h} \omega_{0}$ and $W=100 a$.

Fig. 2

(Color online) (a) Longitudinal conductance $G_{13,13}$ and (b) spin-Hall resistance $R_{13,42}$ as a function of Fermi energy $\mu$ for various values of $\eta$. In the calculation, the system size was set at $L=W=50 a$. Inset is a schematic view of the four-terminal spin-Hall geometry.

Fig. 3

(Color online) Spin-Hall resistance $R_{13,42}$ and longitudinal resistance $R_{13,13}=1 / G_{13,13}$ as a function of normalized SO coupling strength $\eta$. The parameters used in the calculation are $L=W=50 a$ and $\mu=\mathrm{h} \omega_{0}$.

Fig. 4

(Color online) Nonequilibrium spin/charge distribution $\hat{\rho}$ in direct/inverse spin-Hall configurations. Two panels show the density profiles for (a) $\eta=0.1$ and (b) $\eta=1$. The parameters used in the calculation are $L=W=50 a$ and $\mu=\mathrm{h} \omega_{0}$. 


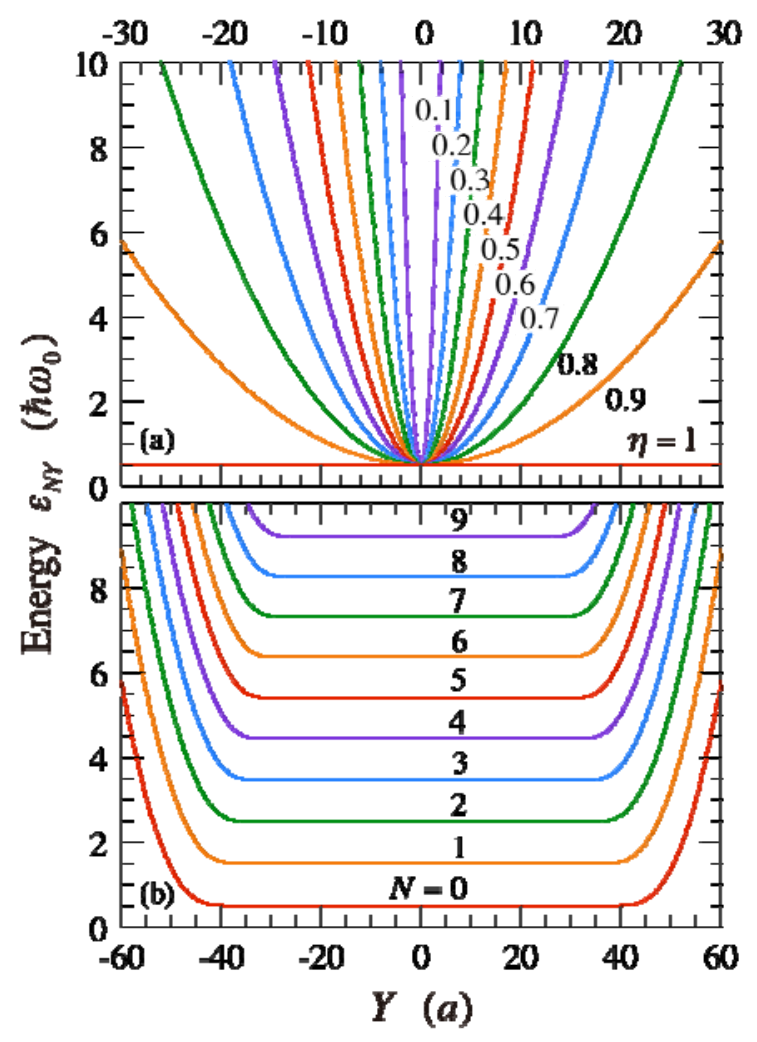

Fig. 1 


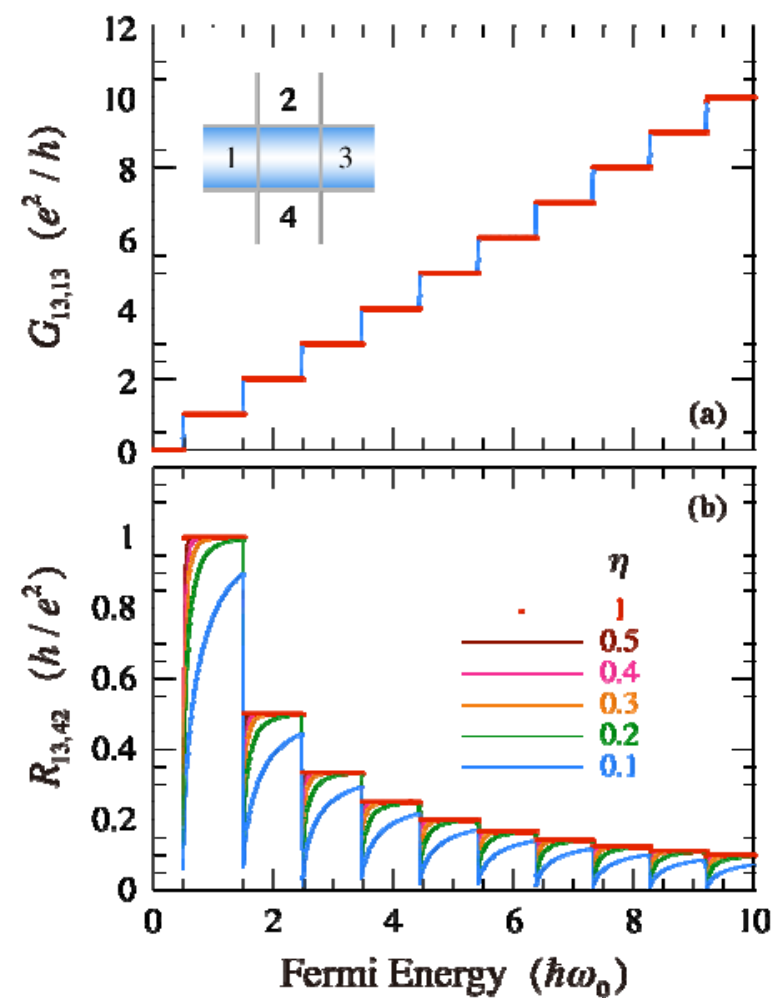

Fig. 2 


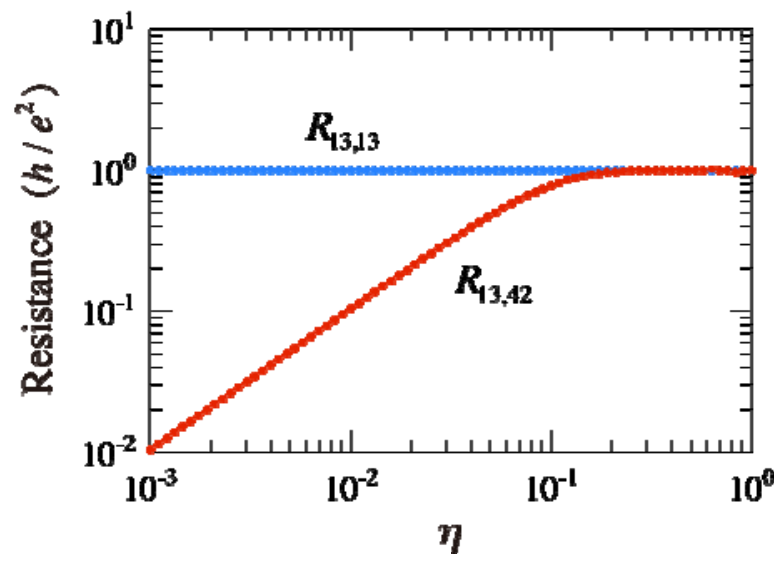

Fig. 3 


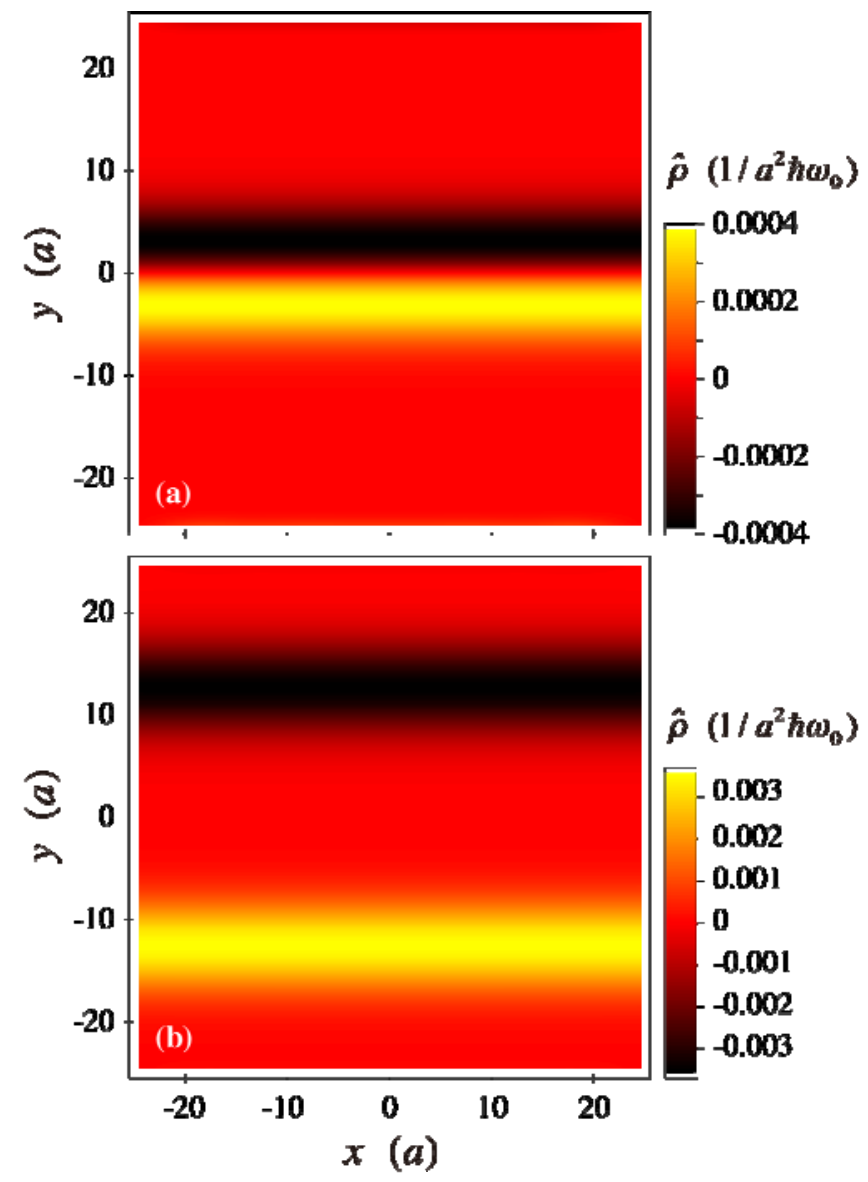

Fig. 4 